\title{
封闭CD28-B7 和OX40-OX40L共刺激通路诱导 异种胰岛移植物耐受的研究 ${ }^{*}$
}

\section{王光明 奉友刚 郝 洁 李爱玲 高 翔 谢蜀生 ${ }^{* *}$}

(北京大学医学部免疫学系, 北京 100083)

\begin{abstract}
摘要 抑制 $\mathrm{T}$ 细胞的活化可以有效延长移植物的存活时间. 研究表明, 在体外混和淋巴细胞反 应体系中分别加入CTLA4Ig和OX40Ig蛋白, 可以强烈地抑制T细胞的增殖. 用链腿佐菌素 (200 $\mathrm{mg} / \mathrm{kg}$ ) 腹腔注射诱导糖尿病小鼠, 在进行胰岛移植的 -1 天, 经尾静脉输注 $5 \times 10^{8} \mathrm{pfu}$ AdCTLA4Ig-IRES-OX40Ig, 胰岛移植物的存活时间明显延长(100.3土 14.94)天, $n=6$, 并对外源性 葡萄糖的刺激反应正常, Th1 型细胞分泌细胞因子的能力明显受到抑制, 表明重组腺病毒 AdCTLA4Ig-IRES-OX40Ig在小鼠体内可以高效地表达CTLA4Ig和OX40Ig分子, 并且诱导对大鼠 胰岛移植物的耐受, 提示其在器官移植耐受的诱导中具有潜在的应用价值.
\end{abstract}

\section{关键词 CD28-B7 OX40-OX40L 链腿佐菌素 重组腺病毒 胰岛移植 | 型糖尿病}

| 型糖尿病是一种自身免疫性疾病, 近年来发 病率快速上升, 已成为严重威胁人类健康的重大疾 病. 目前, 糖尿病尚无有效的治疗方法, 胰岛移植是 治疗糖尿病的理想方法, 但是目前还存在如下主要 问题: ( i ) 胰岛移植物的排斥; (ii) 由于同种胰岛移 植中, 来源于 2 3 个供体的胰岛才能够维持一个移植 受体的需要, 因此供体来源严重不足. 此外, 除大量 的免疫抑制剂的副作用外, 还不可避免的出现免疫 抑制剂对胰岛 $\beta$ 细胞的毒性作用 ${ }^{[1]}$, 因此, 探索异种 移植并使之在体内长期存活是目前移植领域研究的 热点课题. $\mathrm{T}$ 细胞是器官移植排斥的主要效应细胞, $\mathrm{T}$
细胞活化除TCR识别 APC呈递的MHC-肽以外, 还必 需接受由APC提供的共刺激信号才能完成, 因此通 过阻断 $\mathrm{T}$ 细胞活化的共刺激信号以进行异种胰岛移植 是解决目前所存在问题的重要途径.

近来的研究表明, T细胞活化的共刺激信号是一 个多层次序贯表达的复杂网络系统 ${ }^{[2,3]}$, 在器官移植 中封闭一个共刺激通路能够延长移植物的存活时间, 但难以持久. $\mathrm{CD} 28-\mathrm{B} 7$ 是 $\mathrm{T}$ 细胞活化早期的共刺激通 路, 封闭 $\mathrm{CD} 28-\mathrm{B} 7$ 可以有限地延长移植物的存活, 但 不能持久 ${ }^{[2]}$. 最近的研究表明, OX40-OX40L通路提 供的共刺激信号是活化 $\mathrm{T}$ 细胞进一步增殖、分化为 效 
应细胞和记忆细胞所必需的共刺激信号 ${ }^{[3]}$, 是 $\mathrm{T}$ 细胞 介导的免疫应答的关键共刺激信号 ${ }^{[2]}$.

目前已经清楚: ( i ) OX40 的表达不依赖CD28B7 信号, 但是CD28-B7 信号可以促进OX40 的表达; (ii ) CD28-B7 信号和OX40-OX40L信号在时空表达 上具有序贯性, 分别作用于 $\mathrm{T}$ 细胞活化、增殖、分化 的早期和晚期阶段 ${ }^{[4]}$. 因此假设二者在 $\mathrm{T}$ 细胞应答过 程中可能具有协同作用，阻断这两种信号，有可能获 得深度免疫耐受. 我们曾经构建了一个能够同时表 达CTLA4Ig和OX40Ig的真核表达载体，其体外的表 达产物加入混和淋巴细胞反应体系中可以有效地抑 制淋巴细胞的活化增殖, 并显示了明显的协同效应.

本研究中, 在对糖尿病小鼠进行异种胰岛移植 的同时输注能够在体内同时表达 CTLA4Ig 和 OX40Ig 蛋白的重组腺病毒 AdCTLA4Ig-IRES-OX40Ig, 明显 地延长了胰岛移植物的存活时间, 并研究了其可能 机制.

\section{1 材料与方法}

\section{1 实验动物和主要试剂}

雄性Lewis(RT- ${ }^{1}$ )大鼠(供体), 8 16 周龄, 体重 180 300 g; 雄性DA(RT- ${ }^{\mathrm{a}}$ )大鼠(第三者), 8 16 周龄, 体重 180 300 g; 雄性BalB/c(H-2 ${ }^{\mathrm{d}}$ )小鼠(受体), 6 8 周 龄, 体重 16 20 g, 由北京大学医学部实验动物科学 部提供.

链腿佐菌素(Streptozocin, STZ)和 V 型胶原酶购 自Sigma公司; Ficoll400 购自Amersham Pharmacia公 司; ONE TOUCH ${ }^{\circledR}$ II 快速血糖测定仪和血糖检测试 纸购自强生公司; 抗胰岛素多克隆抗体购自 Santa Cruz公司; CTLA4 和OX40 ELISA检测试剂盒购自大 连泛邦化工技术开发有限公司; 其他试剂购自相关 公司或自行配制.

\section{2 重组腺病毒载体的构建及制备}

AdEGFP, AdCTLA4Ig, AdOX40Ig和AdCTLA4IgIRES-OX40Ig的构建、滴度测定见文献[5].

\section{3 胰岛的分离纯化}

参照文献[6]. 经 V 型胶原酶消化、Ficoll400 纯 化和冷Hank's液洗涤后, 取一定的分离物, 用双硫腙
染色, 观察胰岛的形态, 并在倒置显微镜下进行胰岛 计数, 剩余部分悬浮于冷Hank's液中, 密度为 5000 个 胰岛 $/ \mathrm{mL}$.

\section{4 小鼠糖尿病模型的建立}

$\mathrm{BalB} / \mathrm{c}$ 小鼠过夜禁食后, 用柠檬酸缓冲液 $(0.1$ $\mathrm{mol} / \mathrm{L}, \mathrm{pH}$ 4.5)配制成 $10 \mathrm{~g} / \mathrm{L}$ 的链腿佐菌素, 按照 200 $\mathrm{mg} / \mathrm{kg}$ 体重腹腔内注射, $48 \mathrm{~h}$ 后开始剪尾取血, 每周 3 次测定非空腹血糖, 连续 2 次大于 $20 \mathrm{mmol} / \mathrm{L}$ 则定为 实验性糖尿病动物模型.

\section{5 胰岛移植及实验分组}

链脲佐菌素注射 7 天后, 经血糖检测证实为糖尿 病小鼠, 经复合麻醉剂麻醉, 打开腹腔, 暴露左侧肾 脏, 经穿刺把分离的胰岛移植入左侧肾包囊下; 第 3 天测定血糖，在移植的第 1,2 周内，每 2 天测定 1 次， 第 3 周开始, 每周测定 1 次. 非空腹血糖在 $11 \mathrm{mmol} / \mathrm{L}$ 以下为移植物存活, 如果非空腹血糖大于 $11 \mathrm{mmol} / \mathrm{L}$, 需要在后续两天内连续测定血糖, 如果连续 3 次均大 于 $11 \mathrm{mmol} / \mathrm{L}$, 则判定为移植排斥, 排斥时间以第一 次高于 $11 \mathrm{mmol} / \mathrm{L}$ 的时间为准, 结果用胰岛移植物平 均存活时间 (MST) $\bar{x} \pm \mathrm{s}$ 表示. 具体分组如下: 把造模 成功的小鼠随机分为 5 组, 每组 6 只, I 为末作处理 的对照组, II 为AdEGFP处理组, III 为AdCTLA4Ig处 理组, IV 为 AdOX40Ig 处理组, $V$ 为 AdCTLA4IgIRES-OX40Ig处理, 在移植的第- 1 天每只小鼠经尾静 脉给予 $5 \times 10^{8} \mathrm{pfu}$ 腺病毒.

\section{6 小鼠体内 CTLA4Ig 和/或 OX40Ig 表达水平的 检测}

分别于病毒给予当天和第 $1,3,7,10,14,21$ 和 28 天取小鼠尾静脉血, 收集血清, 检测小鼠体内 CTLA4Ig 和/或 OX40Ig 的表达水平.

\section{7 单向混和淋巴细胞反应}

在胰岛移植的第 30 天，取耐受组( III，IV，V组) 小鼠进行混和淋巴细胞反应, 以正常受体品系的 $\mathrm{BalB} / \mathrm{c}$ 小鼠作为对照, 分别测定对供体Lewis大鼠和 第三者DA大鼠的混和淋巴细胞反应. 具体方法如下: 无菌条件下取耐受组及正常受体品系的 $\mathrm{BalB} / \mathrm{c}$ 小鼠 
的脾脏, 以RPMI 1640 培养液制成脾细胞悬液, 台盼 蓝染色计算细胞活性, 用完全RPMI 1640 培养液调整 细胞浓度至 $5 \times 10^{5}$ 个 $/ \mathrm{mL}$, 作为反应细胞. 另取正常 供体Lewis大鼠和第三品系DA大鼠的脾脏, 以相同的 方法制备脾细胞悬液, 用完全RPMI 1640 培养液调整 细胞浓度至 $5 \times 10^{5}$ 个 $/ \mathrm{mL}, 25$ Gy照射作为刺激细胞. 刺激细胞和反应细胞各取 $100 \mu \mathrm{L}$ 加入 96 孔培养板中, 每个组做 9 孔. $37^{\circ} \mathrm{C}$ 孵育 $72 \mathrm{~h}$ 后加入 $0.5 \mu \mathrm{Ci}$ 的 $\mathrm{H}^{3}-\mathrm{TdR}$, 继续孵育 $18 \mathrm{~h}$ 后收集细胞, 测定放射性, 判定结果.

\subsection{RT-PCR 检测小鼠脾细胞 IL-2, IFN- $\gamma$, IL-4 及 IL-10mRNA 表达}

(1) RNA 提取: 按照 Promega 公司提供的 RNA 提取试剂盒的说明进行. PCR 在 $50 \mu \mathrm{L}$ 反应体系中进 行, 小鼠 $\beta$-actin, IL-2, IFN- $\gamma$, IL-4 及 IL-10特异的引 物如下: $\beta$-actin sense 5'-TAA AGA CTA TGC CAA CAC-3'; antisense: 5'-TAA AGC CAT GCC AAA TGT CTC-3'; IL-2 sense 5'-TGC AGC TCG CAT CCT GTC TCA-3'; antisense: 5'-AGA AGG CTA TCC ATC TCC TCA-3'; IFN- $\gamma$ sense: 5'-CTG CAT CTT GGC TTT GC3'; antisense: 5'-CGA CTC CTT TTC CGC TTC CT-3'; IL-4 sense: 5'-TGA CGC ACA GAG CTA TTG ATG G3'; antisense: 5'-ATG ATG CTC TTT AGG CTT TCC AG-3'; IL-10 sense: 5'-ACC TGG TAC AAG TAG TGC CCCC AGA GA-3'; antisense 5'-CAT TGC AGT TGA AGA TGT CAA A-3'.

(2) PCR 产物分析：琼脂糖凝胶电泳. 配制 $1.5 \%$ 的琼脂糖凝胶(含 $0.5 \mu \mathrm{g} / \mathrm{mL} \mathrm{EB}$ ), 取 $5 \mu \mathrm{L}$ PCR 产物进 行电泳, 以 $\beta$-actin 为内参照判断各基因的表达量.

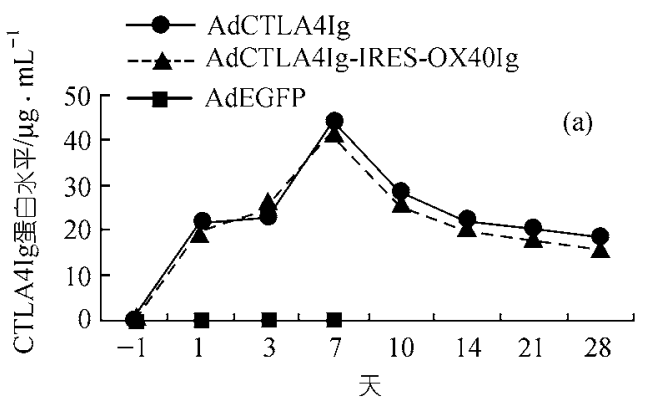

\section{9 胰岛的二次移植}

耐受组( III，IV, V 组)在移植 30 天后经手术摘除 左侧肾脏, 再次进行分别以 Lewis 大鼠和 DA 大鼠为 供体，摘除左侧肾脏的小鼠为受体的胰岛移植，把供 体胰岛移植入右侧肾包囊下, 移植后参照第 1.4 小节 进行血糖监控和移植物排斥的判定.

\subsection{0 葡萄糖耐量实验}

胰岛移植术后 30 天进行葡萄糖耐量实验，同时 以正常 $\mathrm{BalB} / \mathrm{c}$ 小鼠作为对照, 所有受试小鼠均给予 $20 \%$ 葡萄糖 $(1 \mathrm{~g} / \mathrm{kg}$ )腹腔内注射, 并分别于注射前和 注射后 30,60, 90, $120 \mathrm{~min}$ 测定血糖.

\subsection{1 统计学处理}

所有数据均采用 $\overline{\mathrm{x}} \pm \mathrm{s}$ 表示, $t$ 检验法统计学处理.

\section{2 结果}

\section{1 胰岛的分离、纯化结果}

经胰管内胶原酶灌注水浴消化后，每个胰腺获 得 $910 \pm 115$ 个胰岛, 双硫腙染色观察其胰岛形态完整. 经 Ficoll400 密度梯度离心纯化, 获得胰岛 780 999 个, 平均纯度为 $(85.1 \pm 7.1) \%$, 纯化后胰岛回收率为 $(85.7 \pm$ $6.3) \%$ ，与文献报道基本一致.

\section{2 受体小鼠体内 CTLA4Ig 和/或 OX40Ig融合蛋 白表达}

在重组腺病毒给予的当天及后续间隔的数天内, 取小鼠的尾静脉血, ELISA 检测小鼠体内 CTLA4Ig 和 或 OX40Ig 的表达水平, 结果显示(图 $1, n=6$ ), 在 AdCTLA4Ig 给予后 7 天, CTLA4Ig 表达水平达到高

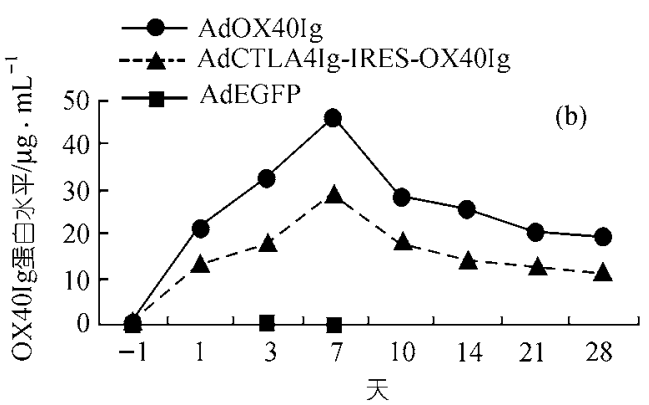

图 1 受体小鼠体内 CTLA4Ig((a)) 和/或 $\mathrm{OX} 40 \operatorname{Ig}((\mathrm{b}))$ 蛋白表达水平 $(n=6)$ 
峰, 随后开始下降, 在第 28 天时仍然维持在较高的 水平 $(18 \mu \mathrm{g} / \mathrm{mL})$; 在 AdOX 40Ig 给予后 7 天, OX40Ig 表 达水平也达到高峰, 随后开始下降, 在第 28 天时仍然 维持在较高的水平 $(19 \mu \mathrm{g} / \mathrm{mL})$; 而在 AdCTLA4IgIRES-OX40Ig 处理组, 虽然 CTLA4Ig 和 OX40Ig 的表 达变化也呈现出与 AdCTLA4Ig 和 AdOX40Ig 处理组 相一致的趋势，但是 OX40Ig 的表达量只达到 AdOx40Ig 处理组的 OX40Ig 表达量的大约 70\%, 而 AdEGFP 处理组未检出 CTLA4Ig 或 OX40Ig 的表达.

\subsection{AdCTLA4Ig-IRES-OX40Ig 基因治疗延长异 基因胰岛移植物存活时间}

胰岛移植物的存活时间见表 1 , 未处理对照组和 AdEGFP组胰岛移植物平均存活时间为 $(6.7 \pm 0.94)$, $(7.0 \pm 1.00)$ 天, 两者间没有统计学意义 $(P>0.05)$; AdCTLA4-Ig组和AdOX40Ig组为(43.2 \pm 4.81$),(67.7 \pm$ $7.74)$, 组间比较有统计学意义 $(P<0.05)$, 与对照组 和 AdEGFP组比较有显著性差异 $(P<0.01)$; AdCTLA4Ig-IRES-OX40Ig组为 $(100.3 \pm 14.94)$ 天, 与上 述 4 个组相比有显著性差异 $(P<0.01)$.

在胰岛移植的第 30 天, 经手术摘除左侧肾脏后, 小鼠的血糖在第二天升高, 达到 $20 \mathrm{mmol} / \mathrm{L}$ 以上, 说 明糖尿病小鼠的血糖降低是由于移植入的胰岛分泌
胰岛素所致. 当以摘除左侧肾脏的小鼠为受体, 分别 以DA大鼠和Lewis大鼠为供体再次进行胰岛移植时, 其胰岛移植物的存活时间见表 2 , 对照组和以DA大 鼠为供体, III $\sim \mathrm{V}$ 组摘除左肾的耐受小鼠为受体的胰 岛移植物平均存活时间分别为 $(6.3 \pm 0.75),(7.3 \pm 0.94)$, (7.7 \pm 1.37$),(6.7 \pm 0.94),(7 \pm 1)$ 天, 组间没有统计学意 义; 而Lewis 大鼠为供体时, $\mathrm{III} \sim \mathrm{V}$ 组的胰岛移植物 存活时间分别为 $(35 \pm 5.71),(40.8 \pm 4.81),(70 \pm 9.0)$ 天, 与对照组和以DA大鼠为供体组相比, 其移植物存活 时间明显延长 $(P<0.01)$, 说明所诱导的免疫耐受是对 Lewis大鼠特异的, 而与第三者无关.

\section{4 受体小鼠脾细胞对供体大鼠脾细胞的混和淋 巴细胞反应(MLR)特异性降低}

取 Lewis 大鼠脾细胞刺激耐受组 $\mathrm{BalB} / \mathrm{c}$ 小鼠脾 细胞, 进行混和淋巴细胞反应, 结果发现: CPM 值明 显降低, 与正常对照组比有显著性差异 $(P<0.01)$, AdCTLA4Ig-IRES-OX40Ig 处理组与 AdCTLA4Ig 或 AdOX40Ig 处理组相比有统计学意义, 由上可见, $\mathrm{BalB} / \mathrm{c}$ 小鼠脾细胞对 Lewis 大鼠脾细胞的应答性降 低, 并且 AdCTLA4Ig-IRES-OX40Ig 组小鼠脾细胞对 Lewis 大鼠脾细胞的应答降低更加显著, 而用 DA 大 鼠的脾细胞刺激时, CPM 值与正常对照无明显差异

表 1 Lewis 大鼠 $\rightarrow \mathrm{BalB} / \mathrm{c}$ 小鼠间胰岛移植物的存活时间

\begin{tabular}{ccccc}
\hline 组 & $n$ & 处理 & 移植物存活时间/天 & 移植物平均存活时间/天 \\
\hline I & 6 & untreated & $8 \times 2,6 \times 4$ & $6.7 \pm 0.94$ \\
II & 6 & AdEGFP & $8 \times 3,6 \times 3$ & $7.0 \pm 1.00$ \\
III & 6 & AdCTLA4Ig & $35,42 \times 3,49 \times 2$ & $43.2 \pm 4.81^{* *}, * * *$ \\
IV & 6 & AdOX40Ig & $63 \times 2,77 \times 2,70,56$ & $67.7 \pm 7.74 * *, * * *$ \\
V & 6 & AdCTLA4Ig-IRES-OX40Ig & $77,91 \times 2,112 \times 2,119$ & $100.3 \pm 14.94 *$ \\
\hline$*$ 与 I IV 组相比, $P<0.01 ; *$ 与 I , II 组相比, $P<0.01 ; * * * ~ I I I, ~ I V$ 组间相比, $P<0.05$ &
\end{tabular}

表 2 Lewis 大鼠和 DA 大鼠 $\rightarrow$ 耐受 $\mathrm{BalB} / \mathrm{c}$ 小鼠间胰岛移植物的存活时间

\begin{tabular}{ccccc}
\hline 供体 & 受体 & $n$ & 移植物存活时间/天 & 移植物平均存活时间/天 \\
\hline Lewis & BalB/c & 6 & $6 \times 5,8 \times 1$ & $6.3 \pm 0.75$ \\
DA & BalB/c & 6 & $6 \times 2,8 \times 4$ & $7.3 \pm 0.94$ \\
Lewis & III & 6 & $28 \times 2,35 \times 2,42 \times 2$ & $35 \pm 5.71^{*}$ \\
DA & III & 6 & $6 \times 2,8 \times 3,10$ & $7.7 \pm 1.37$ \\
Lewis & IV & 6 & $35 \times 2,42 \times 3,49$ & $40.8 \pm 4.81^{*}$ \\
DA & IV & 6 & $6 \times 2,8 \times 4$ & $6.7 \pm 0.94$ \\
Lewis & $\mathrm{V}$ & 6 & $56,63,70 \times 2,77,84$ & $70 \pm 9.0^{*}$ \\
DA & $\mathrm{V}$ & 6 & $6 \times 3,8 \times 3$ & $7 \pm 1$ \\
\hline
\end{tabular}

$*$ 与供体为 $\mathrm{DA}$ 及 Lewis-BalB $/ \mathrm{c}$ 组相比, $P<0.01$ 
$(P>0.05$, 图 2), 说明 $\mathrm{BalB} / \mathrm{c}$ 小鼠脾细胞 MLR 反应 的 CPM 值降低是特异性的, 即针对 Lewis 大鼠脾细 胞的应答受抑制，而对第三者无关个体的应答正常; CTLA4Ig 和 OX40Ig 间显示了较好的协同作用.

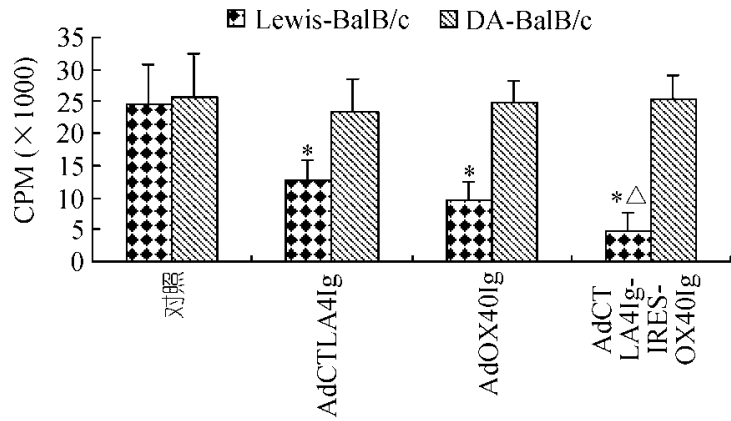

图 2 受体小鼠对供体大鼠脾细胞的 $\operatorname{MLR}(n=5)$ *示 $P<0.01$, 与对照相比; $\triangle$ 示 $P<0.05$, 与耐受的AdCTLA4Ig 和AdOX40Ig相比

\section{5 细胞因子检测}

胰岛移植后 30 天, 取胰岛移植物存活的耐受小 鼠、正常和对照排斥小鼠脾细胞 RNA 作 RT-PCR 以 检测 mRNA 的表达情况，结果发现，耐受小鼠体内 Th1 型细胞因子包括 IL-2 和 IFN- $\gamma$ 的表达较正常小鼠 明显降低，而在排斥小鼠中明显增高，而 Th2 型细胞 因子(包括 IL-10 和 IL-4)的表达与 Th1 细胞因子的表 达情况相反, 而受组其表达增强, 而在排斥组中明显 减弱, 结果见图 3

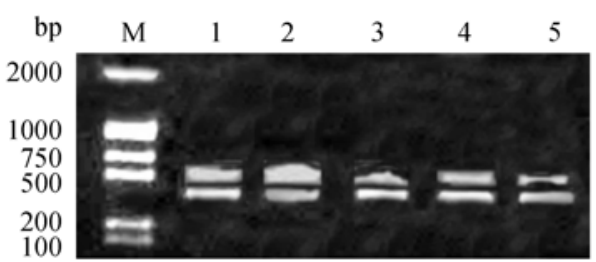

IFN- $\gamma$

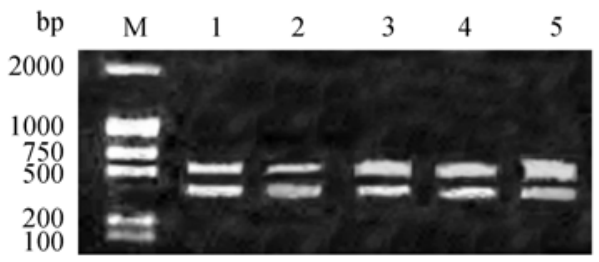

IL-10

\section{6 耐受小鼠对外源性葡萄糖刺激的反应}

在胰岛移植后 30 天, 经腹腔给予外源性葡萄糖 后, 其血糖变化情况见图 4, 即耐受小鼠和正常对照 小鼠的血糖水平急剧升高，在给予葡萄糖后 $30 \mathrm{~min}$, 其血糖水平达到最高值，而在随后的检测中血糖水 平逐渐降低，当在给予外源性葡萄糖 $90 \mathrm{~min}$ 后，其血 糖水平基本恢复到正常水平，耐受组和正常对照组 之间的差异没有统计学意义 $(P>0.05)$.

\section{3 讨论}

CTLA4 是表达在活化T细胞上的一类抑制性受 体，它与表达在 $\mathrm{APC}$ 上的配体 $\mathrm{B} 7$ 分子结合后，可以 抑制 $\mathrm{T}$ 细胞的活化，将其胞外区与改造的人 $\mathrm{IgG1}$ 的 Fc 段融合, 构建的CTLA4Ig融合蛋白显示了结合B7 分 子的高亲和力, 从而可以有效阻断CD28-B7 途径提 供给初始 $\mathrm{T}$ 细胞活化所需的共刺激信号, 诱使 $\mathrm{T}$ 细胞 进入无能，表现为对特定抗原的不应答，因此， CTLA4Ig在诱导移植耐受领域得到广泛的应用 ${ }^{[7 \sim 9]}$. 我们曾用CTLA4Ig体内表达成功地延长了大鼠心脏 及异种胰岛移植物的存活时间，但是不能诱导移植 物的长期耐受 ${ }^{[5,10]}$. 目前认为, 参与 $\mathrm{T}$ 细胞活化的共 刺激信号系统是一个复杂的网络结构 ${ }^{[3]}, \mathrm{CD} 28-\mathrm{B} 7$ 和 CD40-CD40L等共刺激信号途径主要在 $\mathrm{T}$ 细胞活化的 早期发挥作用，但这些通路存在替代途径 ${ }^{[2]}$. 最近

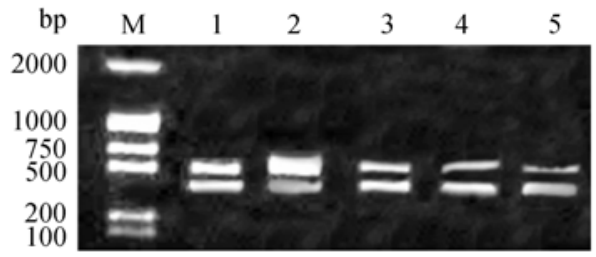

IL-2

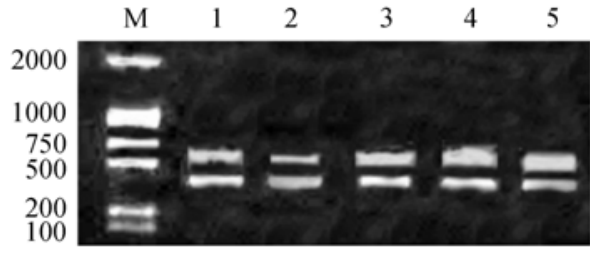

IL-4

图 3 耐受 $\mathrm{BalB} / \mathrm{c}$ 小鼠脾细胞中 $\mathrm{Th} 1 / \mathrm{Th} 2$ 细胞因子 mRNA 的表达

1 示正常小鼠; 2 示移植排斥组; 3 示 AdCTLA4Ig 处理组; 4 示 AdOX40Ig 处理组; 5 示 AdCTLA4Ig-IRES-OX40Ig 处理组 


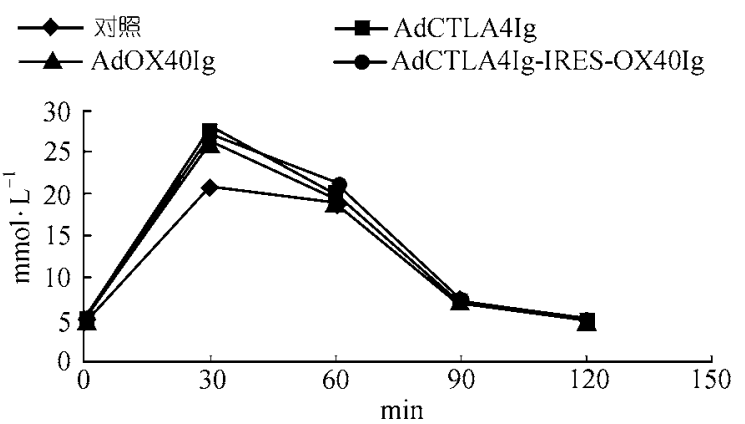

图 4 糖耐量实验检测耐受小鼠对外源性葡萄糖的反应

Demirci等人 ${ }^{[2]}$ 的研究发现, 即使 CD28 和CD40 双基 因敲除小鼠仍然具有接近正常的皮肤排斥能力, 但 是用抗OX40L的抗体封闭OX40-OX40L信号通路后 则皮肤移植物存活明显延长.

目前认为在 $\mathrm{T}$ 细胞活化、增殖、分化的全过程 中, 涉及多个共刺激信号途径, 它们在时空上的相互 协调, 最终导致有效的T细胞应答. OX40(CD134)是 一种跨膜糖蛋白, 属于 TNF受体家族, 在 $\mathrm{T}$ 细胞活化 后 2 3 天高表达, OX40 与 APC上组成性表达的 OX40L结合形成的OX40-OX40L信号通路是T细胞活 化后进一步增殖、分化, 并最终形成效应 $\mathrm{T}$ 细胞(或者 记忆细胞)的关键共刺激信号 ${ }^{[11,12]}$. 已有研究表明, 单独封闭OX40-OX40L信号通路能够延长异基因心 脏移植物的存活时间, 但是不能够获得深度耐受 ${ }^{[13]}$.

本研究利用重组腺病毒介导的基因转移技术, 有效地将CTLA4Ig基因和OX40Ig基因导入到受体小 鼠体内, 使其在体内分别有效表达 CTLA4Ig和 OX40Ig蛋白, 发挥作用. 研究表明, 在AdCTLA4IgIRES-OX40Ig给予后 4 周, 受体小鼠血清中CTLA4Ig 和OX40Ig蛋白的浓度仍然维持在 16 和 $13 \mu \mathrm{g} / \mathrm{mL}$ 的高 水平，保证了抑制 $\mathrm{T}$ 细胞活化有效浓度 ${ }^{[14]}$, 从而延长 胰岛移植物的存活时间, 在该研究中, AdCTLA4IgIRES-OX40Ig 组的胰岛移植物存活时间为(100.3 \pm 14.94)天, 而 AdCTLA4Ig 组和 AdOX40Ig 组分别为 $(43.2 \pm 4.81),(67.7 \pm 7.74)$ 天, 表明通过重组腺病毒 AdCTLA4Ig-IRES-OX40Ig介导的基因转移所产生的 CTLA4Ig和OX40Ig蛋白在胰岛移植物的存活上显示 了强烈的协同作用. 混和淋巴细胞反应结果显示耐 受小鼠脾细胞对供体来源的Lewis大鼠脾细胞的刺激
反应性降低，而对第三者DA大鼠脾细胞刺激仍然具 有正常的反应性. 糖耐量实验结果显示耐受小鼠对 外源性葡萄糖的刺激具有与正常小鼠一致的反应能 力. 当切除耐受小鼠左侧肾脏后, 耐受小鼠的血糖在 第二天就升高, 并且在摘除的肾包囊下的胰岛移植 物具有分泌胰岛素的功能. 在耐受小鼠切除左侧肾 脏的同时进行二次胰岛移植，当胰岛来源于与第一 次移植相同的供体时, 胰岛移植物仍然能够长期存 活, 而当胰岛移植物来源于第三者(DA大鼠)时, 其胰 岛移植物在移植的第 6 8 天被排斥, 说明经重组腺病 毒 AdCTLA4Ig-IRES-OX40Ig诱导的免疫耐受是对 Lewis大鼠特异的, 耐受动物对其他抗原仍然具有正 常的反应能力, 同时还说明耐受小鼠血糖的降低是 由于移植在肾包囊下的胰岛移植物分泌的胰岛素所 致. 用RT-PCR检测Th1 型细胞因子(包括IL-2 和IFN- $\gamma$ ) 和Th2 型细胞因子(包括IL-10 和IL-4)的表达时发现, 在耐受小鼠中 Th1 型细胞因子较正常小鼠明显降低, 而Th2 型细胞因子的表达较正常小鼠的表达升高, 说 明诱导的移植耐受可能与 Th1/Th2 型细胞偏移有关.

以上结果说明经重组腺病毒AdCTLA4Ig-IRESOX40Ig介导的基因转移可以在受体小鼠体内高表达 CTLA4Ig和OX40Ig蛋白, 从而有效地抑制了 T细胞的 活化，延长了胰岛移植物的存活时间. 在该重组腺病 毒中, 由于引入了核糖体进入位点(IRES)序列 ${ }^{[15]}$, 使 得CTLA4Ig和OX40Ig蛋白可以同时表达, CTLA4Ig与 APCs上的B7 分子结合, 有效地阻断了 $\mathrm{T}$ 细胞活化所 需的CD28-B7 共刺激途径, 而逃避了 CTLA4Ig阻断 的部分活化 $\mathrm{T}$ 细胞, 由于 $\mathrm{OX} 40 \mathrm{Ig}$ 与 $\mathrm{APCs}$ 上的 $\mathrm{OX} 40 \mathrm{~L}$ 结合而阻断了它们进一步分化所需的OX 40-OX40L 共刺激信号, 使 $\mathrm{T}$ 细胞不能够进一步分化为效应细胞 和记忆细胞 ${ }^{[16]}$, 从而有效地阻断了 $\mathrm{T}$ 细胞应答的发 生.

本研究成功构建双表达 CTLA4Ig 和 OX40Ig 的 重组腺病毒 AdCTLA4Ig-IRES-OX40Ig, 将其用于大 鼠异种胰岛移植，并证实 CD28-B7 和 OX40-OX40L 信号通路同时阻断具有较好的协同作用和免疫抑制 效果, 有一定的理论意义. 该重组腺病毒还可以应用 于其他实体器官移植的实验研究, 有一定的实验研 究价值, 但是要用于临床, 还有很长的路要走. 


\section{参考文献}

1 Berney T, Buhler L H, Majno P, et al. Immunosuppression for pancreatic islet transplantation. Transplantation Proceeding, 2004, 36(s2): 362s 368s[DOI]

2 Demirci G, Amanullah F, Kewalaramani R, et al. Critical role of OX40 in CD28 and CD154-independent rejection. J Immunology, 2004, 172: 1691 1698

3 Patrick H. Still a long way to go before immune tolerance. Transplantation, 2002, 73: s43 s44

4 Tania $\mathrm{H}$ W. TNF/TNFR family members in costimulation of $\mathrm{T}$ cell response. Annu Rev Immunol, 2005, 23: 23 68[DOI]

5 Yongzhu Jin, Qingyin Zhang, Jie Hao, et al. Simultaneous administration of a low-dose mixture of donor bone marrow and splenocyte plus adenovirus containing the CTLA4Ig gene result in stable mixed chimerism and long-term survival of cardiac allograft in rats. Immunology, 2003, 110: 275 286[DOI]

6 Chaib E, Papalois A, Brons I G, et al. Isogenic islet transplantation on the rat liver(method for isolation and purification of the langerhans islets). Arg Gastroenterol, 2000, 37(1): 44 51

7 Yang Z, Rostami S, Koeberlein B, et al. Cardiac allograft tolerance induced by intra-arterial infusion of recombinant adenoviral CTLA4Ig. Transplantation, 1999, 67(12): 1517 1523[DOI]

8 Feng S, Quickel R R, Hollister-Lock J, et al. Prolonged xenograft survival of islets infected with small doses of adenovirus expressing CTLA4Ig. Transplantation, 1999, 67(12): 1607 1613[DOI]

9 Ariyan C, Salvalaggio P, Fecteau S, et al. Cutting edge: Trans- plantation tolerance through enhanced CTLA-4 expression. The Journal of Immunology, 2003, 171: 5673 5677

10 Y-Z Jin, S-S Xie. Bicistronic Adenovirus-mediated gene transfer of CTLA4Ig gene and CD40Ig gene result in indefinite survival of islet xenograft. Transplantatoin Proceedings, 2003, 35: 3165 3166[DOI]

11 Peter L. Role of OX40 signals in coordinating CD4 T cell selection, migration, and cytokine differentiation in $\mathrm{T}$ helper(Th) 1 and Th2 cells. J Exp Med, 2000, 191: 201 205[DOI]

12 Prell R A, Evans D E, Thalhofer C, et al. OX40-mediated memory $\mathrm{T}$ cell generation is TNF receptor-associated factor 2 dependent. Journal Immunology, 2003, 171: 5997 6005

13 Curry A J, Chikwe J, Smith X G, et al. OX40(CD134) blockade inhibits the co-stimulatory cascade and promotes heart allograft survival. Transplantation, 2004, 78: 807 814[DOI]

14 Weinberg A D, Wemann K W, Funatake C, et al. Blocking OX-40/ OX-40 ligand interaction in vitro and in vivo leads to decreased $\mathrm{T}$ cell function and amelioration of experimental allergic encephalomyelitis. The Journal of Immunology, 1999, 162: 1818 1826

15 Mizuguchi H, Xu Z L, Akiko I W, et al. IRES-dependent second gene expression is significantly lower than cap-dependent first gene expression in a bicistronic vector. Molecular Therapy, 2000, 1: 376 382[DOI]

16 Prell R A, Evans D E, Thalhofer C, et al. OX40-mediated memory $\mathrm{T}$ cell generation is TNF receptor-associated factor 2 dependent. The Journal of Immunology, 2003, 171: 5997 6005 